\title{
A IMPLICAÇÃO DAS ATITUDES PARENTAIS NO USO DE DROGAS NA ADOLESCÊNCIA
}

\author{
Fernanda Cerutti ${ }^{1}$; Sérgio de Paula Ramos ${ }^{2}$; Irani Iracema de Lima Argimon ${ }^{1 *}$ \\ ${ }^{1}$ Pontifícia Universidade Católica do Rio Grande do Sul-Brasil, ${ }^{2}$ Fundação de Incentivo a Pesquisa em Álcool e Drogas-Brasil
}

Recibido, octubre $7 / 2014$

Concepto de evaluación, mayo 12/2015

Aceptado, mayo 25/2015
Referencia:Cerutti, F., Ramos, S. P. \& Argimon, I.I.L. (2015). A implicação das atitudes parentais no uso de drogas na adolescencia. Acta Colombiana de Psicología, 18(2), 73-181. DOI: 10.14718/ACP.2015.18.2.15

Resumo

Este estudo transversal quantitativo objetivou analisar a relação entre a percepção do adolescente sobre as atitudes parentais e sua tendência ao uso de substâncias psicoativas. Participaram 487 adolescentes brasileiros. Para análise estatística foi utilizado o Modelo Logístico Multinomial, sobre o qual se aplicou o método Stepwise para seleção de variáveis. O tabaco, o álcool e a maconha foram relacionados com as variáveis de maior influência no uso, abuso ou dependência. Verificou-se que um pai pouco afetivo, aumenta a chance de o filho ser dependente de tabaco $(p=0.001)$. A mãe sendo pouco afetiva tem maior possibilidade de ter um filho dependente do tabaco $(p=0.001)$. O controle do pai é um fator protetivo para a experimentação do tabaco $(p=0.023)$ e para a dependência da maconha $(p=0.042)$. A relação entre o uso de drogas na adolescência e a percepção que o jovem tem das atitudes de seus pais ficou evidenciada.

Palavras-chave: atitudes parentais, adolescência, drogadição

\section{INFLUENCIA DE LAS ACTITUDES DE LOS PADRES EN EL CONSUMO DE DROGAS EN LA ADOLESCENCIA}

\begin{abstract}
Resumen
El objetivo de este estudio transversal cuantitativo fue analizar la relación entre la percepción del adolescente sobre su tendencia al uso de sustancias psicoactivas y las actitudes de sus padres. Participaron 487 adolescentes brasileños. Para el análisis estadístico se utilizó el Modelo Logístico Multinomial, en el que se aplicaba el método Stepwise para la selección de variables. El tabaco, el alcohol y la marihuana se relacionaron con las variables de mayor influencia en el uso, abuso y dependencia. Se encontró que un padre poco cariñoso aumenta la posibilidad de que el niño sea dependiente del tabaco $(p=$ $0.001)$, al igual que una madre poco afectuosa tiene más probabilidades de tener un hijo dependiente del tabaco $(p=0.001)$. El control del padre es un factor protector para la experimentación del tabaco $(p=0.023)$ y para la dependencia de la marihuana $(p=0.042)$. Se puso de relieve la relación entre el consumo de las drogas en la adolescencia y la percepción que los jóvenes tienen de las actitudes de sus padres.

Palabras clave: actitudes de los padres, adolescencia abuso de drogas.
\end{abstract}

\section{INFLUENCE OF PARENTAL ATTITUDES ON SUBSTANCE CONSUMPTION IN ADOLESCENCE}

\begin{abstract}
The aim of this quantitative cross-sectional study was to analyze the relationship between the perception of adolescents about their tendency to use psychoactive substances and their parents' attitudes. 487 Brazilian adolescents participated. For statistical analysis the Multinomial Logit Model was used, where the Stepwise method for selecting variables was applied. Tobacco, alcohol and marijuana were related to the variables of greatest influence on the use, abuse and dependence of these substances. Findings showed that an unloving father increases the possibility that the child becomes tobacco dependent $(\mathrm{p}=$
\end{abstract}

\footnotetext{
* Av. Ipiranga, 6681, Partenon, Porto Alegre - RS, 90619-900, Brasil, 55 (51) 3320-3500 - Ramal 4986. argimoni@pucrs.br Os autores agradecem aos alunos da graduação em psicologia da PUCRS e da URI/FW pela colaboração na coleta dos dados. Ressaltam o apoio financeiro recebido pela CAPES e CNPQ.
} 
0.001). An unloving mother is most likely to have a tobacco dependent child $(\mathrm{p}=0.001)$. Father's control is a protective factor for experimenting with tobacco smoking $(p=0.023)$ and for marijuana dependence $(p=0.042)$. The relationship between substance consumption in adolescence and the perception young people have about their parents' attitudes was emphasized. Key words: parental attitudes, adolescence, drug abuse

\section{INTRODUÇÃO}

Os comportamentos arriscados do adolescente, que implicam em uma vulnerabilidade elevada aos efeitos das substâncias psicoativas, vêm sendo explicados através do desenvolvimento ainda em curso do lobo córtex pré-frontal (Winters, 2008). Essa imaturidade cerebral somada aos dados sobre o consumo de substâncias psicoativas por adolescentes apontam que a prevenção do uso de drogas nessa fase ainda é um tema que necessita ser sempre debatido.

Nos Estados Unidos, o projeto Monitoring the Future (MTF) apresenta dados sobre o uso de drogas na adolescência assinalando que $12 \%$ de adolescentes com idades entre 13 e 14 anos, $34 \%$ com idades entre 15 e 16 anos já haviam se embriagado pelo menos uma vez na vida. Quanto ao uso de tabaco, um percentual de $15 \%$ de adolescentes com idades de 13 a 14 anos referem ter usado tabaco e $5 \%$ relataram fumar nos últimos 30 dias. Daqueles com 15 a 16 anos, $26 \%$ disseram ter experimentado cigarros, e $9,1 \%$ relataram fumar nos últimos 30 dias. $O$ uso na vida de maconha abrange $36 \%$ com 15 a 16 anos, $17 \%$ com idades entre 13 e 14 anos. No último ano, 30\% com 15 a 16 anos e $13 \%$ de 13 a 14 anos fizeram uso de maconha. E no último mês, o uso de maconha foi entre $18 \%$ daqueles com 15 a 16 anos e 7\% com 13 a 14 anos (Johnston, O’Malley, Bachman, Schulenberg \& Miech, 2013).

$\mathrm{Na}$ Europa, de acordo com dados mais recentes levantados pelo European School Survey Project on Alcohol and Other Drugs (ESPAD) em 2011, estima-se que um em cada quatro adolescentes europeus de 15 a 16 anos já experimentou uma droga ilícita, sendo que $24 \%$ desses referem consumo regular de cannabis. A respeito do uso de drogas lícitas, $19 \%$ dos jovens entrevistados teriam fumado um ou mais cigarros de tabaco diariamente no mês anterior e $4 \%$ deles afirmaram já ter fumado mais de 10 cigarros de tabaco por dia. Com relação ao álcool, $57 \%$ dos estudantes fizeram uso de álcool no mês anterior, sendo que $20 \%$ se intoxicaram com a bebida pelo menos uma vez no referido período (Hibell et al., 2012).

A realidade brasileira sobre uso de drogas na adolescência foi investigada através de uma amostra nacionalmente representativa de 17.371 estudantes do ensino médio de 789 escolas públicas e privadas de todas as capitais brasileiras. O estudo revelou que $82 \%$ dos participantes já haviam usado álcool, dentre os quais, $11 \%$ experimentaram álcool precocemente, antes dos 12 anos (Sanchez et al., 2013).
Outro levantamento nacional sobre uso de drogas (II LENAD), que contou com uma amostra randomizada de 4.607 pessoas representantes de todos os estados brasileiros, com idades a partir dos 14 anos, sendo que 1.157 eram adolescentes, destaca que aproximadamente $600 \mathrm{mil}$ adolescentes ( $4 \%$ da população) já fizeram uso de maconha pelo menos uma vez na vida e 470 mil ( $3 \%$ da população) usaram no último ano. A prevalência de uso de cocaína incide em 2\% da população (Laranjeira, 2014).

A precocidade do uso de substâncias psicoativas também está sendo associada com os riscos no desenvolvimento do adolescente. Os jovens que usaram álcool precocemente têm mais chances de abusar de álcool do que aqueles que iniciaram o uso mais tardiamente. Além disso, são mais propensos a já terem usado tabaco ou outra droga ilegal. $\mathrm{O}$ uso precoce está associado à percepção de uma falta de punição por parte dos pais para o uso de álcool e ao fato dos cuidadores consumirem drogas (Sanchez et al., 2013).

Em se tratando dos fatores que influenciam o uso de drogas na adolescência, o uso de substâncias psicoativas pelos pais é uma variável que se relaciona positivamente com prejuízos no desenvolvimento do filho. Adolescentes que convivem com pais dependentes de drogas têm um risco aumentado de desenvolver um distúrbio psicopatológico no decorrer da adolescência (Marmorstein, Iacono \& McGue, 2009), podendo favorecer o uso de drogas.

O tabagismo dos pais foi associado com comportamentos de risco à saúde em adolescentes, tais como uma maior probabilidade de fumar, uso frequente de álcool e uso de drogas ilícitas (Virtanen et al., 2009). Ademais, o histórico de Transtorno de Uso de Substâncias Ilícitas dos pais está associado com o desenvolvimento mais precoce de Transtorno de Uso de Substâncias Ilícitas pelo filho adolescente com diagnóstico de Depressão Maior (Gorka, Shankman, Seeley \& Lewinsohn, 2013).

A influência dos pais na busca pelo uso de drogas pelo filho também ocorre através de outros fatores. A orientação e o monitoramento são comportamentos parentais de proteção. Adolescentes que fazem parte de famílias cujos pais são mais tolerantes e favoráveis ao uso de drogas têm maiores chances de consumir substâncias psicoativas do que aqueles que recebem orientação e são desencorajados ao uso (Miller \& Plant, 2010). O monitoramento dos pais também diminui os riscos do uso de substâncias pesadas por jovens com uma infância com vivências difíceis (Fallu et al., 2010). 
O vínculo é outro fator relevante nessa problemática. Um vínculo frágil entre mãe e filho está associado com o desenvolvimento de traços de personalidade que favorecem o uso de drogas, que está associado com a escolha por parceiros também usuários de drogas, sendo esses fatores de grande risco para o desenvolvimento do Transtorno por Uso de Substâncias (Brook, J. S., Brook, Zhang \& Cohen, 2009). Adolescentes com uma ligação fraca com os familiares têm maior chance de usar drogas (Moura, Sanchez \& Noto, 2010). Em contrapartida, a prevenção e diminuição do uso estão associadas com um maior envolvimento e proximidade dos pais (Winters, Fahnhorst, Botzet, Lee \& Lalone, 2012).

O objetivo deste estudo foi analisar a relação entre a percepção do adolescente sobre as atitudes parentais e sua tendência de uso de substâncias psicoativas, através de modelos de gravidade do envolvimento com as drogas. Especificamente, buscou-se verificar a influência do uso de álcool ou de tabaco pelos familiares no uso de drogas na adolescência.

\section{MÉTODO}

\section{Participantes}

Estudo transversal, quantitativo, que contou com uma amostra escolhida por conveniência de 487 adolescentes de um município do interior do Rio Grande do Sul, Brasil. Neste município há um predomínio de moradores descentes de imigrantes italianos e alemães. Os participantes frequentavam os quatro últimos anos do ensino fundamental e os três anos do ensino médio em cinco escolas do meio urbano, duas particulares e três públicas.

\section{Instrumentos}

Os instrumentos utilizados incluíam três questionários autoaplicáveis. O primeiro foi a Ficha de Dados Sociodemográficos elaborada para o estudo, composta por questões que investigaram variáveis sociodemográficas como idade, sexo, escolaridade, dados sobre os pais, hábitos familiares quanto ao uso de drogas lícitas e acesso e uso dos participantes quanto às drogas ilícitas.

O segundo questionário é o Alcohol, Smoking and Substance Involvement Screening Test (ASSIST), desenvolvido sob a coordenação da Organização Mundial de Saúde, que tem como finalidade detectar uso de álcool, tabaco e outras substâncias psicoativas, por meio de oito questões estruturadas referentes ao uso de nove classes de substâncias: tabaco, álcool, maconha, cocaína, estimulantes, sedativos, inalantes, alucinógenos e opioides. Essas questões abordam a frequência do uso, na vida e nos últimos três meses, e a relação do indivíduo com as substâncias (World Health Organization, 2002; Humeniuk, et. al., 2008). A confiabilidade do instrumento, testado no estudo de Henrique, Micheli, de Lacerda, R. B., de Lacerda e Formigoni (2004), apresenta alfa de Cronbach de 0,80 para álcool, 0,79 para maconha e 0,81 para cocaína.

O terceiro instrumento é o Parental Bonding Instrument-PBI (Parker, Tupling \& Brown, 1979). Questionário autoaplicável que mede a contribuição dos comportamentos dos pais no desenvolvimento de seus filhos, em que o participante deve responder sobre o comportamento do pai e da mãe, separadamente, até os seus 16 anos. Foi elaborado baseado em análises fatoriais de 114 itens pertinentes a qualidades parentais destacados da literatura. Contém 25 afirmativas com pontuações distribuídas tipo escala Likert (0 a 3) e é dividido em duas escalas: Afeto/Cuidado (12 itens) e Superproteção/Controle (13 itens). A pontuação máxima para as escalas de afeto e controle é de 36 e 39 pontos, respectivamente. Assim, um escore alto na escala Afeto/ Cuidado indica uma percepção de carinho e proximidade, enquanto que altos escores na escala de Superproteção/ Controle representam percepção de excessiva proteção, vigilância e a presença de infantilização. Os pontos de corte do estudo original apresentam-se, para as mães, na escala de afeto, em 27,0, e, na escala de proteção, 13,5. Para os pais, os pontos de corte são 24,0 na escala de afeto e 12,5 na escala de proteção.

No instrumento, também, são contemplados quatro tipos de vínculos: Cuidado Ótimo (controle baixo/afeto alto), Controle Afetivo (afeto alto/controle alto), Controle sem Afeto (controle alto/afeto baixo) e Negligente (afeto baixo/controle baixo). A consistência interna do estudo original apresentou coeficiente Alfa de Cronbach de 0,88 para escala de afeto e 0,74 para escala de proteção (Parker, 1989). No estudo de Teodoro, Benetti, Schwartz e Mônego (2010), que analisou as propriedades psicométricas do PBI em uma amostra de adultos jovens da região metropolitana de Porto Alegre-RS, o coeficiente de alfa de Cronbach para a escala de afeto materno foi 0,91 e controle materno 0,87 , na versão paterna, foi 0,91 e 0,85 para afeto e controle respectivamente. A versão do instrumento utilizada nesse estudo foi adaptada transculturalmente por Hauck et al. (2006).

\section{Procedimento}

Esta pesquisa foi aprovada pelo Comitê de Ética da PUCRS-Brasil e registrada sob o número CAAE 10896512.0.0000.5336. A coleta de dados, que foi desenvolvida no mês de abril de 2013, foi conduzida por aplicadores treinados em sessões coletivas realizadas nas salas de aula das escolas participantes. A direção das escolas 
e os alunos e seus pais que aceitaram participar da coleta receberam informações sobre os objetivos da pesquisa e procedimentos, respeitando as questões éticas. Os Termos de Consentimento Livre e Esclarecido e de Assentimento foram entregues em duas vias.

\section{Análise de dados}

A análise dos dados contemplou análises descritivas (frequências) e o cálculo do coeficiente alfa de Cronbach, utilizado para estimar a confiabilidade dos questionários aplicados. Para avaliar a gravidade de envolvimento com cada droga, (0: Não uso [baseline], 1:Uso, 2: Abuso e 3: Dependência), utilizou-se o Modelo Logístico Multinomial Nominal, ajustado com auxílio do método Stepwise para a seleção automática de variáveis (Faraway, 2006). O procedimento, inicialmente, cruzou as categorias não uso, uso, abuso e dependência das drogas (álcool, tabaco e maconha) com as variáveis sexo, idade, escolaridade da mãe e do pai, situação de vida da mãe e do pai, se algum familiar fumava tabaco ou ingeria bebida alcoólica diariamente, se o participante já havia utilizado alguma droga ilícita, e também com os quatro tipos de vínculos e os dois estilos parentais. Posteriormente, foram trabalhados alguns modelos ajustados para cada droga quanto às quatro categorias de gravidade de envolvimento e às variáveis que melhor se adequavam.

\section{RESULTADOS}

Primeiramente, serão apresentadas as frequências de dados sociodemográficos, uso de drogas lícitas e ilícitas e as características parentais. Em seguida, os coeficientes de alfa de Cronbach encontrados. Por último, os modelos de gravidade do uso de álcool, maconha e tabaco ajustados com variáveis associadas pela literatura ao uso de drogas na adolescência.

A amostra foi composta por $55,6 \%$ participantes do sexo feminino. A distribuição das idades por faixa etária apresenta a maior frequência (44,3\%) entre os adolescentes com 15 e 16 anos. A maioria dos estudantes $(32,2 \%)$ estava frequentando o $1^{\circ}$ ano do Ensino Médio, e 85,4\% estudavam em escola pública.

Quando questionados sobre terem usado alguma droga ilícita, 4,7\% dos participantes afirmaram já terem consumido. Desses, 0,6\% usaram substâncias ilícitas naquela semana, no final de semana anterior e na semana precedente, e $2,6 \%$ no último mês. Se desejassem comprar alguma droga proibida, $34,9 \%$ saberiam onde encontrar.

Quanto às frequências para a experimentação de cada uma das nove classes de drogas estudadas, no que diz respeito às substâncias lícitas, $15,0 \%$ já fizeram ou fazem uso de tabaco, e $81,7 \%$ já experimentaram o álcool. No que se refere a experimentação de drogas lícitas, 7,0\% já experimentaram maconha e 3,9\% cocaína. Para as demais classes, 1,4\%dos adolescentes já usaram anfetaminas, 2,5\% inalantes, 3,5\% hipnóticos, 3,9\% alucinógenos e 1,2\% experimentaram opioides.

Em se tratando dos tipos de vínculos dos pais percebidos pelos adolescentes, $19,9 \%$ dos pais e $22,0 \%$ mães são cuidadosos e afetuosos de forma suficiente com seus filhos, $34,2 \%$ dos pais e $40,0 \%$ das mães são afetivos, porém com elevado controle, $32,9 \%$ dos pais e $32,4 \%$ mães são controladores e pouco afetivos e, por último, $13,0 \%$ pais e $5,6 \%$ mães foram considerados negligentes por seus filhos. A Figura 1 apresenta a proporção dos tipos de vínculos entre pais e mães.

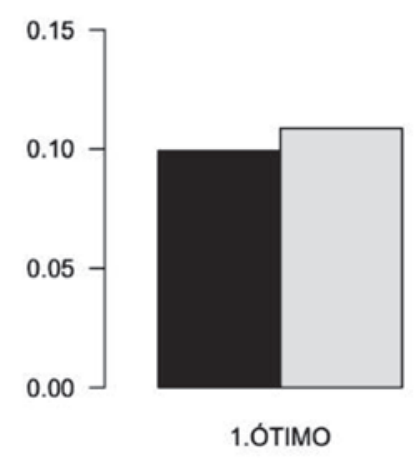

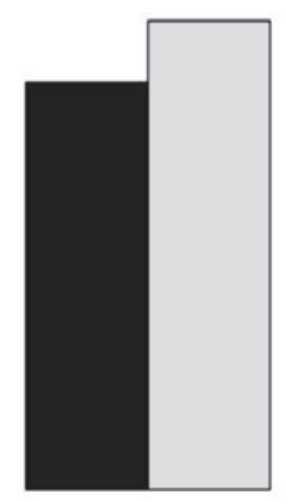

2.AFETO.COM.CONTROLE

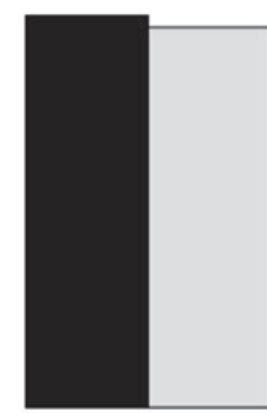

3.CONTROLE SEM AFETO
- Pai

Mãe

Vínculo

Figura 1. Proporções do Tipo de Vínculo de Pais e Mães. 
Os coeficientes de alfa de Cronbach encontrados foram analisados considerando-se o intervalo de confiança (Bootstrap) de $95 \%$. No questionário ASSIST, o coeficiente de alfa de Cronbach é 0,940 (IC $=0,854-0,967)$, para o PBI na versão da mãe, 0,806 na escala do Afeto (IC $=0,780$ $0,827)$ e 0,699 na escala do Controle ( $\mathrm{IC}=0,650-0,737)$ e na versão paterna 0,868 na escala do Afeto $(\mathrm{IC}=0,847$ $0,884)$ e 0,729 na escala do Controle ( $\mathrm{IC}=0,687-0,766)$.

Na Tabela 1, estão publicados os modelos para as drogas tabaco, álcool e maconha, ajustados com as variáveis que melhor se adequaram em relação ao uso, abuso e dependência de cada substância, todos em relação ao não uso (baseline).

O modelo do tabaco considerado ajustou a variável idade com valores não significativos para $p \leq 0,05$ e $p \leq 0,01$, porém o Critério de Informação de Akaike mostrou-se significativo
$(\mathrm{AIC}=500.5586)$. Embora como uma tendência, em relação a variável idade, pode-se estimar que quanto maior a idade do participante, maior a probabilidade deste ser abusador ou dependente, e menor a chance de experimentar ou fazer apenas uso da substância. Este adolescente, tendo alguém que faça uso de bebida alcoólica diariamente em sua casa, tem maior chance de ser um tabagista e, se o mesmo ainda tiver experimentado ou feito uso de alguma droga ilícita, tem maior possibilidade de abusar do tabaco.

Quanto aos estilos parentais, o modelo sugere que um adolescente com uma mãe pouco afetiva tem maior possibilidade de ser dependente da droga, assim como um pai pouco afetivo tem mais chance de ter um filho dependente de tabaco. Porém, se for mais controlador, tem menor possibilidade de que seu filho experimente tabaco.

Tabela 1.

Modelo de Gravidade do Envolvimento com o Tabaco Ajustado com as Variáveis: Idade, Uso Diário de Álcool por Familiares, Uso de Drogas Ilícitas, Afeto Baixo da Mãe e do Pai, e Controle do Pai

\begin{tabular}{llcccc}
\hline \multicolumn{1}{c}{ Variável } & Categoria & Coeficientes & Erros padrão & T & $\mathrm{p}$ \\
\hline \multirow{3}{*}{ Idade } & Uso & -0.043 & 0.153 & -0.282 & 0.389 \\
& Abuso & 0.158 & 0.109 & 1.448 & 0.074 \\
& Dependência & 0.211 & 0.365 & 0.578 & 0.282 \\
\multirow{2}{*}{ Uso diário de álcool em casa } & Uso & 0.882 & 0.505 & 1.746 & $* 0.041$ \\
& Abuso & 0.796 & 0.356 & 2.236 & $* 0.013$ \\
& Dependência & 1.018 & 1.053 & 0.966 & 0.167 \\
Uso de drogas ilícitas & Uso & 0.382 & 1.105 & 0.346 & 0.365 \\
& Abuso & 2.265 & 0.502 & 4.509 & $* * 0.001$ \\
Afeto Mãe (Baixo) & Dependência & 1.454 & 1.275 & 1.141 & 0.127 \\
& Uso & -0.103 & 0.510 & -0.203 & 0.420 \\
Afeto Pai (Baixo) & Abuso & -0.311 & 0.364 & -0.855 & 0.196 \\
& Dependência & 11.106 & 2.014 & 5.515 & $* * 0.001$ \\
& Uso & 0.548 & 0.492 & 1.114 & 0.133 \\
Controle Pai & Abuso & 0.461 & 0.350 & 1.314 & 0.094 \\
& Dependência & 11.914 & 2.013 & 5.918 & $* * 0.001$ \\
& Uso & -0.074 & 0.037 & -1.993 & $* 0.023$ \\
& Abuso & 0.033 & 0.025 & 1.301 & 0.097 \\
& Dependência & 0.021 & 0.087 & 0.242 & 0.404 \\
\hline
\end{tabular}

Nota. $\mathrm{AIC}=500.5586 ; p \leq 0,05^{*} ; p \leq 0,01^{* *}$ 
Tabela 2.

Modelo de Gravidade de Envolvimento com o Álcool Ajustado com as Variáveis: Idade, Uso Diário de Álcool por Familiares, Uso de Drogas Ilícitas e Afeto do Pai

\begin{tabular}{llcccc}
\hline \multicolumn{1}{c}{ Variável } & Categoria & Coeficientes & Erros padrão & $\mathrm{T}$ & $\mathrm{P}$ \\
\hline \multirow{3}{*}{ Idade } & Uso & 0.102 & 0.073 & 1.406 & 0.080 \\
& Abuso & 0.290 & 0.092 & 3.141 & $* * 0.001$ \\
& Dependência & 0.212 & 0.195 & 1.084 & 0.139 \\
\multirow{4}{*}{ Uso diário de álcool em casa } & Uso & 0.633 & 0.363 & 1.744 & $* 0.041$ \\
& Abuso & 1.671 & 0.377 & 4.426 & $* * 0.001$ \\
& Dependência & 2.563 & 0.643 & 3.986 & $* * 0.001$ \\
\multirow{3}{*}{ Uso de drogas ilícitas } & Uso & 1.807 & 1.052 & 1.718 & $* 0.043$ \\
& Abuso & 1.750 & 1.094 & 1.599 & $* 0.055$ \\
\multirow{3}{*}{ Afeto Pai } & Dependência & 3.313 & 1.325 & 2.500 & $* * 0.006$ \\
& Uso & 0.016 & 0.013 & 1.194 & 0.116 \\
& Abuso & -0.027 & 0.016 & -1.698 & $* 0.045$ \\
& Dependência & 0.073 & 0.039 & 1.850 & $* 0.032$ \\
\hline
\end{tabular}

Nota. $\mathrm{AIC}=500.5586 ; p \leq 0,05^{*} ; p \leq 0,01 * *$

Com relação ao modelo ajustado para o álcool, salienta-se que quanto maior a idade do participante, maior a chance de ele ser um abusador da substância. Se o adolescente tem algum parente que ingere bebida alcoólica diariamente, tem maior possibilidade de abusar e desenvolver o alcoolismo.
E se já fez uso ou é usuário de alguma droga ilícita, tem grande chance de ser alcoolista. Quanto ao estilo parental, filhos de pais mais afetivos apresentam menos chances abusar de álcool, porém têm mais possibilidade de serem dependentes dessa droga lícita.

Tabela 3.

Modelo de Gravidade de Envolvimento com a Maconha Ajustado com as Variáveis: Sexo, Uso de Drogas Ilícitas e Controle do Pai

\begin{tabular}{|c|c|c|c|c|c|}
\hline Variável & Categoria & Coeficientes & Erros padrão & $\mathrm{t}$ & $\mathrm{P}$ \\
\hline \multirow{3}{*}{ Sexo } & Uso & 0.813 & 0.768 & 1.059 & 0.145 \\
\hline & Abuso & 0.331 & 0.415 & 0.797 & 0.213 \\
\hline & Dependência & 9.865 & 0.714 & 13.809 & $* * 0.001$ \\
\hline \multirow{3}{*}{ Uso de drogas ilícitas } & Uso & 1.542 & 1.117 & 1.381 & 0.084 \\
\hline & Abuso & 2.732 & 0.499 & 5.472 & $* * 0.001$ \\
\hline & Dependência & 4.102 & 1.668 & 2.460 & $* * 0.007$ \\
\hline \multirow{3}{*}{ Controle Pai } & Uso & 0.017 & 0.058 & 0.298 & 0.383 \\
\hline & Abuso & -0.013 & 0.033 & -0.397 & 0.346 \\
\hline & Dependência & -0.373 & 0.216 & -1.726 & $* 0.042$ \\
\hline
\end{tabular}

Nota. $\mathrm{AIC}=500.5586 ; p \leq 0,05^{*} ; p \leq 0,01^{* *}$ 
No que diz respeito ao modelo que foi ajustado para a maconha, ressalta-se que o participante, sendo do sexo masculino, tem mais chance de ser dependente da substância e, se já experimentou ou faz uso de alguma droga ilícita, apresenta maior possibilidade de abusar e ser dependente da maconha. Já se o usuário tiver um pai controlador, tem mais chance de não ser dependente da droga.

\section{DISCUSSÃO}

Os dados descritivos apontam que o álcool é a substância mais usada entre os participantes. Fato que deve ser destacado, pois o álcool é uma droga lícita que causa muitos danos ao jovem. Pode servir de porta de entrada para outras drogas, como o tabaco, a maconha e outras substâncias ilícitas (Kirby \& Barry, 2012). Além de colocar o jovem em situações de agressividade, pois os adolescentes que usam álcool têm maior probabilidade do que os abstêmios de se envolver em eventos como lutas físicas, sofrer agressões e ter pensamentos de violência autodirigida (Marmorstein et al., 2009).

Os resultados demonstram ainda que o uso de drogas ilícitas pelo adolescente aumenta a chance do mesmo abusar do tabaco, ser dependente do álcool e abusar e depender da maconha. Um estudo que relacionou o uso de maconha, droga ilícita que comumente recebe aceitação social, aos casos de adolescentes que chegavam até a emergência pediátrica com evidências de uso abusivo de álcool, identificou que entre esses, os que também faziam uso de maconha, tinham altas taxas de tabagismo e de consumo excessivo de álcool (Chun et al., 2010).

Ademais, o uso diário de bebida alcoólica pelos familiares aumenta a possibilidade de que o adolescente seja dependente de tabaco e do álcool. Um estudo longitudinal, que objetivou analisar a influência do alcoolismo paterno no abuso de substâncias dos jovens, encontrou associação entre o uso de substâncias psicoativas pelos filhos que tinham pais dependentes de álcool (Ohannessian \& Hesselbrock, 2008). Outro estudo longitudinal, que propunha examinar a contribuição dos fatores de risco de pais no uso de drogas dos filhos, constatou que os fatores de risco dos pais (uso de drogas e conflitos) têm forte associação com o aumento do uso de substâncias pelos jovens (Elkington, Bauermeister \& Zimmerman, 2011).

Quanto aos estilos parentais, os achados sobre o afeto paterno, o apresentam como um fator importante de risco para o uso de drogas na adolescência. Se o pai é pouco afetivo, tem mais chance de o seu filho ser dependente de tabaco e de o filho abusar de álcool. No entanto, se for mais afetivo, aumenta a chance de dependência de álcool do filho. Assim, pode-se entender que o distanciamento afetivo dos pais ou uma permissividade maior, confundida por um excesso de afeto, podem predizer um baixo controle do uso das substâncias e uma maior impulsividade (PatockPeckham \& Morgan-Lopez, 2006).

O controle do pai é um fator protetor para a experimentação do tabaco e para a dependência da maconha. E nesse estudo não foi apontado como fator de risco. Indicando que possivelmente os adolescentes relacionaram mais o controle paterno com o monitoramento do que com autoritarismo, pois a maioria dos participantes considerou que seus pais eram afetivos, porém mantinham o controle. O controle e o monitoramento parental foram também relacionados como fatores de proteção para o uso de drogas na adolescência em outros estudos (Kopak, Chia-Chen Chen, Haas \& Gillmore, 2012; Piko \& Kóvacs, 2010; Pokhrel, Unger, Wagner, Ritt-Olson \& Sussmann, 2008; Poulin \& Denault, 2012).

$\mathrm{O}$ afeto materno se evidenciou somente como fator de risco e não como de proteção, e apenas em relação ao tabaco. Uma mãe pouco afetiva tem maior chance de ter um filho dependente do tabaco. Sobre a relação mãefilho, um fraco vínculo entre a mãe e o filho prediz traços de personalidade no filho que favorecem o uso de drogas (Brook et al., 2009). O sofrimento psicológico das mães pode ser outro fator implicado no afeto materno, que, em um estudo, relacionou-se com o consumo excessivo de álcool e uso de drogas ilícitas entre adolescentes (HermanStahl et al., 2008).

A observação dos achados deve considerar as limitações do estudo que dizem respeito ao número de questões que os participantes deviam preencher e aos temas de conteúdo difícil que os instrumentos propunham levantar. Somados a esses dois aspectos, está a fase da vida dos participantes da amostra, que também pode ser uma limitação, uma vez que se tratam de adolescentes que têm como características certo grau de impulsividade, tempo menor de tolerância para permanecer na mesma atividade e muitos questionamentos a respeito de suas características e dificuldades.

Outro ponto a ser destacado é a escolha dos instrumentos. Quanto ao ASSIST, que apontou o risco do uso de algumas classes de drogas, a análise dos dados através da pontuação sugerida não prevê a diferenciação com o "não uso", ou seja, de participantes abstinentes. Para esse estudo, verificou-se pertinente essa diferenciação. Além disso, sugere-se para novos estudos a aplicação de questionários com o mesmo objetivo, porém adaptados especificamente ao público adolescente e pré-adolescente.

Em relação ao PBI, destaca-se que pelo fato do mesmo ter sido elaborado e proposto há mais de duas décadas, baseou-se em preceitos sobre o cuidado parental que 
tendia a ser mais afetivo e não ser rígido como visto nas gerações anteriores. Entretanto, o que está sendo revisto, principalmente na última década, é a necessidade de monitoramento e da participação mais direta dos pais. Aspecto não contemplado diretamente no questionário.

Ressalta-se, em relação à alta prevalência do uso do álcool, que o uso dessa droga deve estar prioritariamente contemplado em pesquisas que abordem a temática da adolescência. Parece importante salientar que essas informações devem alcançar não somente os jovens, mas, da mesma forma, seus familiares e cuidadores, pois ficou evidenciada a repercussão do consumo de bebidas alcoólicas em casa e o uso de drogas pelo adolescente.

Ainda sobre o acesso aos pais, a partir desses resultados, ressalta-se a importância de pesquisas que priorizem o acompanhamento e a orientação dos pais de adolescentes, tanto no que diz respeito ao bom prognóstico e adesão no tratamento de filhos usuários de substâncias, quanto na prevenção do uso de drogas na adolescência.

\section{REFERÊNCIAS}

Brook, J. S., Brook, D. W., Zhang, C. \& Cohen, P. (2009). Pathways from Adolescent Parent-Child Conflict to Substance Use Disorders in the Fourth Decade of Life. The American Journal on Addictions, 18, 235-242. doi:10.1080/10550490902786793

Chun, T. H., Spirito, A., Hernández, L., Fairlie, A. M., SindelarManning, H., Eaton, C. A. \& Lewander, W. J. (2010). The Significance of Marijuana Use among Alcohol-using Adolescent Emergency Department Patients. Society for Academic Emergency Medicine, 17, 63-71. doi:10.1111/j.15532712.2009.00615.x

Elkington, K. S., Bauermeister, J. A. \& Zimmerman, M. A. (2011). Do parents and peers matter? A prospective socio-ecological examination of substance use and sexual risk among African American youth. Journal of Adolescence, 34, 1035-1047. doi:10.1016/j.adolescence.2010.11.004

Fallu, J. S., Janosz, M., Brière, F. N., Descheneaux, A., Vitaro, F. \& Tremblay, R. E. (2010). Preventing disruptive boys from becoming heavy substance users during adolescence: A longitudinal study of familial and peer-related protective factors. Addictive Behaviors, 35, 1074-1082. doi:10.1016/j. addbeh.2010.07.008

Faraway, J. J. (2006). Extending the Linear Model with R: Generalized Linear, Mixed Effects and Nonparametric Regression Models. New York, NY: Boca Raton London.

Gorka, S. M., Shankman, S. A., Seeley, J. R. \& Lewinsohn, P. M. (2013). The moderating effect of parental illicit substance use disorders on the relation between adolescent depression and subsequent illicit substance use disorders. Drug and Alcohol Dependence, 128, 1-7. doi:10.1016/j.drugalcdep.2012.07.011.
Hauck, S., Schestatsky, S., Terra, L., Knijnik, L., Sanchez, P. \& Ceitlin, L. H. F. (2006). Adaptação transcultural para o português brasileiro do Parental Bonding Instrument (PBI). Revista de Psiquiatria do Rio Grande do Sul, (28)2, 162-168. doi: 10.1590/S0101-81082006000200008

Henrique, I. F. S., Micheli, D., de Lacerda, R. B., de Lacerda, L. A. \& Formigoni, M. L. O. S. (2004). Validação da versão brasileira do teste de triagem do envolvimento com álcool, cigarro e outras substâncias (ASSIST). Revista da Associação Médica Brasileira, 50(2), 199-206. doi: 10.1590/S010442302004000200039

Herman-Stahl, M. A., Ashley, O. S., Penne, M. A., Bauman, K. E., Williams, J., Sanchez, R. P., ...Gfroerer, J. C. (2008). Moderation and Mediation in the Relationship between Mothers' or Fathers' Serious Psychological Distress and Adolescent Substance Use: Findings from a National Sample. Journal of Adolescent Health, 43,141-150. doi: 10.1016/j. jadohealth.2008.01.010

Hibell, B., Guttormsson, U., Ahlström, S., Balakireva, O., Bjarnason, T., Kokkevi, A. \& Kraus, L. (2012). The 2011 ESPAD Report Substance Use Among Students in 36 European Countries. Sweden, Stockholm: The Swedish Council for Information on Alcohol and Other Drugs (CAN).

Humeniuk, R., Ali, R., Babor, T. F., Farrell, M., Formigoni, M L., Jittiwutikarn, J., . . Simon, S. (2008). Validation of the Alcohol Smoking and Substance Involvement Screening Test (ASSIST). Addiction 103(6), 1039-1047. doi:10.1111/j.13600443.2007.02114.x

Johnston, L. D., O’Malley, P. M., Bachman, J. G., Schulenberg, J. E. \& Miech, R. A., (2013). Monitoring the Future national survey results on drug use, 1975-2013 (vol. I): Secondary school students. Ann Arbor, Michigan: Institute for Social Research, The University of Michigan.

Kirby, T. \& Barry, A. E. (2012). Alcohol as a gateway drug: a study of US 12th graders. Journal of School Health, 82(8), 371-379. doi: 10.1111/j.1746-1561.2012.00712.x

Kopak, A. M., Chia-Chen Chen, A., Haas, S. A. \& Gillmore, M. R. (2012). The importance of family factors to protect against substance use related problems among Mexican heritage and White youth. Drug and Alcohol Dependence 124, 34-41. doi:10.1016/j.drugalcdep.2011.12.004

Laranjeira, R. (Org.). (2014). II Levantamento Nacional de Álcool e Drogas (LENAD) -2012. São Paulo: Instituto Nacional de Políticas Públicas do Álcool e Outras Drogas (INPAD).

Marmorstein, N. R., Iacono, W. G. \& McGue, M. (2009). Alcohol and illicit drug dependence among parents: associations with offspring externalizing disorders. Psychological Medicine, 39(1), 149-155. doi:10.1017/S00 33291708003085

Miller, P. \& Plant, M. (2010). Parental guidance about drinking: Relationship with teenage psychoactive substance use. Journal of Adolescence, 33, 55-68. doi:10.1016/j.adolescence. 2009.05.011

Moura, Y. G., Sanchez, Z. M. \& Noto A. R. (2010). Diversity of Contexts in Drug Use among Street Adolescents. Qualitative Health Research, 20(9), 1241-1253. 
Ohannessian, C. M. \& Hesselbrock, V. M. (2008). Paternal Alcoholism and Youth Substance Abuse: The Indirect Effects of Negative Affect, Conduct Problems, and Risk Taking. Journal of Adolescent Health, 42, 198-200. doi:10.1016/j. jadohealth.2007.08.025.

Parker, G. (1989). The parental bonding instrument: Psychometric properties reviewed. Psychiatry Developed, (7)4, 317-335.

Parker, G., Tupling, H. \& Brown, L. B. (1979). Parental Bonding Instrument (PBI). British Journal of Medical Psychology, 52, 1-10.

Patock-Peckham, J. A. \& Morgan-Lopez, A. A. (2006). College drinking behaviors: Mediational links between parenting styles, impulse control, and alcohol-related outcomes. Psychology of Addictive Behaviors, 20(2), 117-125. doi: 10.1037/0893164X.20.2.117.

Piko, B. F. \& Kovács, E. (2010). Do parents and school matter? Protective factors for adolescent substance use. Addictive Behaviors, 35, 53-56. doi:10.1016/j.addbeh.2009.08.004.

Pokhrel, P., Unger, J. B., Wagner, K. D., Ritt-Olson, A. \& Sussman, S. (2008). Effects of Parental Monitoring, Parent-Child Communication, and Parents' Expectation of the Child's Acculturation on the Substance Use Behaviors of Urban, Hispanic Adolescents. Journal of Ethnicity in Substance Abuse, 7(2), 200-213. doi:10.1080/15332640802055665.

Poulin, F. \& Denault, A. S. (2012). Other-Sex Friendships as a Mediator between Parental Monitoring and Substance Use in Girls and Boys. Journal of Youth Adolescence, 41(11), 1488-1501. doi: 10.1007/s10964-012-9770-y.

Sanchez, Z. M., Santos, M. G. R., Pereira, A. P. D., Napo, S. A., Carlini, C., Carlini, E. A. \& Martins, S. S. (2013). Childhood Alcohol Use May Predict Adolescent Binge Drinking: A Multivariate Analysis among Adolescents in Brazil. The Journal of Pediatrics, 163, 363-368. doi: 10.1016/j.jpeds.2013.01.029.

Teodoro, M. L. M., Benetti, S. P. C., Schwartz, C. B. \& Mônego, B. G. (2010). Propriedades psicométricas do Parental Bonding Instrument e associação com funcionamento familiar. Avaliação Psicológica, 9(2), 243-251.

Virtanen, M., Pietikäinen, M., Kivimäki, M., Luopa, P., Jokela, J., Elovainio, M. \& Vahtera, J. (2009). Contribution of parental and school personnel smoking to health risk behaviours among Finnish adolescents. BMC Public Health, 9, 382-390. doi:10.1186/1471-2458-9-382.

Winters, K. C., Fahnhorst, T., Botzet, A., Lee, S. \& Lalone, B. (2012). Brief intervention for drug-abusing adolescents in a school setting: Outcomes and mediating factors. Journal of Substance Abuse Treatment, 42(3), 279-288. doi: 10.1016/j. jsat.2011.08.005.

Winters, K. C. (2008). Adolescent Brain Development and Drug Abuse. Minnesota: The Mentor Foundation.

World Health Organization. (2002). Working Group. The Alcohol, Smoking and Substance Involvement Screening Test (ASSIST): development, reliability and feasibility. Addiction, 97, 1183-1194. doi:10.1046/j.1360-0443.2002.00185.x 